M. OZAWA

KODAI MATH. J.

1 (1978), 339-35̄3

\title{
ON UNIQUELY FACTORIZABLE MEROMORPHIC FUNCTIONS
}

\author{
By Mitsuru Ozawa
}

1. Although our knowledges on the factorization theory are increasing day by day, the theory is still in the infancy, since almost all the fundamental problems remain unsettled. We are currently accustomed to make use of the word "prime", however the unique factorizability into prime factors does not hold in general. Further there is no general theorem which gives the unicity or the non-unicity of factorization. Up to the present time we only know few theorems concerning the unique factorizability, which are applicable to quite special classes of entire or meromorphic functions. This paper, which may be considered as a continuation of our earlier paper [8], is also concerned with the unique factorizability theorems of special types. Our results are the following theorems.

THEOREM 1. Let $f(z)$ be a prime entire function of finite order. Assume that $f^{\prime}(z)$ has infinitely many zeros and $f(z)=c, f^{\prime}(z)=0$ have only finitely many common roots for every constant $c$. Let $p$ be a prime integer. Then $F(z)=f\left(z^{p}\right)$ is uniquely factorizable.

THEOREM 2. Let $f(z)$ be a prime entire function of finte order. Assume that, for a certain constant $A, f(z)=A$ has at least one non-zero simple root and at most finitely many simple roots except for infinitely many multiple roots all of whose orders are equal to $\mu$. Assume further that $f(z)=c, f^{\prime}(z)=0$ have only finitely many common roots for every $c \neq A$. Let $p$ be a prime number satisfying $(p, \mu)=1$. Then $F(z)=f\left(z^{p}\right)$ is unquely factorizable.

These two theorems can be proved by making use of our earlier theorems in [6], [7]. Therefore we can construct corresponding theorems for an entire function of infinite order. We shall not give them here. The function $z^{p} \exp \left(p z^{p}\right)$ shows that theorem 1 does not hold if we omit the main assumption in theorem 1 .

THEOREM 3. Let $f_{0}(z)$ be the entire function defined by

$$
\begin{aligned}
& f_{0}(z)=\Pi_{1}\left(e^{z}\right) \Pi_{2}\left(e^{-z}\right), \\
& \Pi_{1}(w)=\prod_{n=1}^{\infty} E\left(\frac{w}{a_{n}}, p_{n}\right)^{\nu_{n},}
\end{aligned}
$$

Received June 9, 1977. 


$$
\Pi_{2}(w)=\prod_{n=1}^{\infty}\left(1-\frac{w}{b_{n}}\right)^{\mu_{n}}
$$

where $E(x, p)$ is the Weierstrass primary factor

$$
\begin{gathered}
(1-x) \exp \left(x+\frac{1}{2} x^{2}+\cdots+\frac{1}{p} x^{p}\right), \\
\sum_{n=1}^{\infty} \frac{\mu_{n}}{\left|b_{n}\right|^{\delta}}<\infty
\end{gathered}
$$

for a positive $\delta<1$ and $\nu_{n}, \mu_{n}$ are prime numbers satisfying $3 \leqq \nu_{n}, 3 \leqq \mu_{n}, \nu_{n}<\nu_{n+1}$, $\mu_{n}<\mu_{n+1}, \mu_{n} \neq \nu_{m}$ for every pair $(n, m)$. Let $F(z)$ be $f_{0}(z) \exp f_{0}(z)$. Then $F(z)$ is uniquely factorizable.

In our earlier paper [6] we had proved that $f_{0}(z)$ in this theorem 3 is a periodic entire function which is prime.

THEOREM 4. Let $f_{0}(z)$ be

$$
\begin{aligned}
& \left(e^{z}-1\right) \exp \left(e_{n}(z)+e^{-z}\right), \\
& e_{n}(z)=\exp \left(e_{n-1},(z)\right), e_{1}(z)=e^{z} .
\end{aligned}
$$

Then $F(z)=f_{0}(z) \exp f_{0}(z)$ is uniquely factorizable.

THEOREM 5. Let $f_{0}(w)$ be $\pi_{1}(w) / \pi_{2}(w)$, where

$$
\begin{aligned}
& \pi_{1}(w)=\prod_{\jmath=1}^{\infty}\left(1-\frac{w}{a_{\jmath}}\right)^{\nu \jmath}, \\
& \pi_{2}(w)=\prod_{\jmath=1}^{\infty}\left(1-\frac{w}{b_{\jmath}}\right)^{\mu_{\jmath}}
\end{aligned}
$$

with $a_{\jmath} \neq b_{k}$ for every pair $(j, k)$ and

$$
\sum_{j=1}^{\infty} \frac{\nu_{j}}{\left|a_{j}\right|^{\grave{\delta}}}<\infty, \quad \sum_{j=1}^{\infty} \frac{\mu_{j}}{\left|b_{j}\right|^{\grave{\delta}}}<\infty
$$

for an arbitrary positive number $\delta$ and prime numbers $\nu_{\jmath}, \mu_{\jmath}$ such that $3 \leqq \nu_{\jmath}, 3 \leqq \mu_{\jmath}$, $\nu_{j}<\nu_{j+1}, \mu_{j}<\mu_{j+1}, \nu_{j} \neq \mu_{k}$. Let $g_{0}(z)$ be a prime entrre function such that $f_{0}\left(g_{0}(z)\right)$ is of order less than one. Then $f_{0}\left(g_{0}(z)\right)$ is uniquely factorizable.

The following theorem is an extension of Theorem 1 in [8].

THEOREM 6. Let $F(z)$ be $g_{1}(z) e^{H\left(g_{1}(z)\right)}$, where $H(w)$ is a polynomial and $g_{1}(z)$ us a prime transcendental entire function of finte order having infinitely many zeros $\left\{z_{n}\right\}$, which lie in the sector 


$$
|\pi-\arg z| \leqq \begin{cases}\frac{\pi}{2(\rho+\varepsilon)}, & \rho \geqq 1 \\ \frac{\pi}{2(1+\varepsilon)}, & \rho<1 .\end{cases}
$$

$\varepsilon>0$ and $\rho$ is the order of $g_{1}(z)$. Then $F(z)$ is uniquely factorizable.

2. Proof of Theorem 1. Let us consider

This is just

$$
\left\{\begin{array}{l}
F(z)=c \\
F^{\prime}(z)=0 .
\end{array}\right.
$$

$$
\left\{\begin{array}{l}
f\left(z^{p}\right)=c \\
f^{\prime}\left(z^{p}\right) p z^{p-1}=0 .
\end{array}\right.
$$

Hence there are only finitely many common roots. Evidently $F^{\prime}(z)=0$ has infinitely many roots and $F(z)$ is of finite order. Hence by our earlier theorem in [6] $F(z)$ is left-prime in entire sense. Let $F(z)$ be $f_{1}\left(g_{1}(z)\right)$. If $f_{1}$ and $g_{1}$ are entire, then $f_{1}$ is transcendental and $g_{1}$ is a polynomial. Evidently $F(z)$ is p-ply symmetric and asymptotically $n$-ply symmetric, where $n=\operatorname{deg} g_{1}(z)$. Hence $n=p r$ with a positive integer $r$. We have

$$
f_{1}\left(g_{1}(z)\right)=F(z)=F(\omega z)=f_{1}\left(g_{1}(\omega z)\right)
$$

with every $\omega$ satisfying $\omega^{p}=1$. Let $g_{1}(z)$ be $a_{n} z^{n}+\cdots+a_{1} z+a_{0}$. Then by an elegant theorem due to Baker-Gross [1] the following two cases are possible: 1 ) $\left.g_{1}(\omega z)=\lambda g_{1}(z)+\beta, 2\right) g_{1}(z)=s(z)^{2}+k, g_{1}(\omega z)=(s(z)+c)^{2}+k$ for some polynomial $s(z)$.

Case 1). In this case

$$
a_{n} \omega^{n} z^{n}+\cdots+a_{1} \omega z+a_{0}=\lambda\left(a_{n} z^{n}+\cdots+a_{1} z+a_{0}\right)+\beta .
$$

Therefore $\lambda=1, \omega^{n}=1$ and $a_{j}=0$ for $\jmath \neq p t, t=0,1, \cdots, r$. Hence

$$
g_{1}(z)=a_{p r} z^{p r}+a_{p(r-1)} z^{p(r-1)}+\cdots+a_{p} z^{p}+a_{0} .
$$

Let us put $w=z^{p}$. Then

$$
f(w)=f_{1}\left(\sum_{j=0}^{r} a_{p j} w^{j}\right)
$$

Since $f(w)$ is prime, $r$ should be equal to 1 . Hence $g_{1}(z)=a_{p} z^{p}+a_{0}$ and $f(w)=$ $f_{1}\left(a_{p} w+a_{0}\right)$. Hence we have two equivalent factorizations of $F(z)$.

Case 2). In this case

$$
\left(g_{1}(z)-g_{1}(\omega z)+c^{2}\right)^{2}=4 c^{2}\left(g_{1}(z)-k\right),
$$


that is,

$$
\begin{gathered}
\left\{a_{n}\left(1-\omega^{n}\right) z^{n}+a_{n-1}\left(1-\omega^{n-1}\right) z^{n-1}+\cdots+a_{1}(1-\omega) z+c^{2}\right\}^{2} \\
=4 c^{2}\left(a_{n} z^{n}+a_{n-1} z^{n-1}+\cdots+a_{1} z+a_{0}-k\right) .
\end{gathered}
$$

Hence $a_{j}\left(1-\omega^{j}\right)=0$ for $j=[n / 2]+1, \cdots, n$. If $n$ is odd, $c=0$. This gives $g_{1}(\omega z)$ $=g_{1}(z)$. This case reduces to case 1$)$. If $n$ is even,

$$
a_{n / 2}^{2}\left(1-\omega^{n / 2}\right)^{2}=4 c^{2} a_{n} .
$$

Since $n=p r$ is even, $r$ is even. Hence $\omega^{n / 2}=\left(\omega^{p}\right)^{r / 2}=1$. Thus $c=0$. Again this case reduces to case 1 ).

Assume that $f_{1}$ is transcendental meromorphic (not entire) and $g_{1}$ is transcendental entire. Then

$$
\begin{array}{cl}
f_{1}(w)=\frac{f^{*}(w)}{\left(w-w_{1}\right)^{n}}, & g_{1}(z)=w_{1}+A e^{M(z)}, \quad M(0)=0, \\
f^{*}\left(w_{1}\right) \neq 0 .
\end{array}
$$

Hence

$$
F(z)=f^{*}\left(w_{1}+A e^{M(z)}\right) A^{-n} e^{-n M(z)} .
$$

$M(z)$ is a polynomial. Let $U(w)$ be

$$
A^{-n} f^{*}\left(w_{1}+A e^{w}\right) e^{-n w}
$$

Then

$$
F(z)=f\left(z^{p}\right)=U(M(z)) .
$$

Here $U$ is entire and $M$ is a polynomial. Hence by the result already proved $M(z)$ should be equal to $a z^{p}$. Let $W$ be $z^{p}$. Then

$$
f(W)=U\left(a_{p} W\right)=\frac{f^{*}\left(w_{1}+A e^{a W}\right)}{A^{n} e^{n a W}} .
$$

This contradicts the primeness of $f(W)$.

Other cases are very easy to handle. We have the desired result.

The proof of Theorem 2 is quite similar as in Theorem 1.

3. Proof of Theorem 3. Let $F(z)$ be $f(g(z))$. Let $f$ and $g$ be transcendental entire. Then we can make use of the same reasoning as in the proof of the primeness of $f_{0}(z)$ in [9] and we can prove that $f$ has only one zero $w_{1}$. Then

$$
\begin{gathered}
f(w)=A\left(w-w_{1}\right) e^{L(w)}, \quad L(0)=0, \\
A\left(g(z)-w_{1}\right)=B f_{0}(z) e^{M(z)}, \quad M(0)=0 .
\end{gathered}
$$

Hence 
Evidently

$$
\begin{gathered}
f(g(z))=B f_{0}(z) e^{M(z)} e^{L(g(z)),} \\
f_{0}(z)=D+M(z)+L(g(z)), \\
g(z)=C f_{0}(z) e^{M(z)}+w_{1} .
\end{gathered}
$$

$$
\begin{gathered}
g(z+2 \pi i)-g(z)=C f_{0}(z) e^{M(z)}\left(e^{-N(z)}-1\right), \\
L(g(z+2 \pi i))-L(g(z))=-M(z+2 \pi i)+M(z) \equiv N(z) .
\end{gathered}
$$

If $N(z) \not \equiv$ const, we have

$$
\bar{N}\left(r, 1, e^{N(z)}\right) \leqq N(r, 0, N(z)),
$$

which gives a contradiction. If $N(z) \equiv a, a$ should be equal to zero. Hence $M(z)=h_{1}\left(e^{z}\right), g(z)=h_{2}\left(e^{z}\right)$ with one-valued regular functions $h_{1}(w)$ and $h_{2}(w)$ in $0<$ $|w|<\infty$. Hence putting $w=e^{z}$ we have

$$
\begin{aligned}
& \Pi_{1}(w) \Pi_{2}\left(\frac{1}{w}\right)=D+h_{1}(w)+L\left(h_{2}(w)\right), \\
& h_{2}(w)=C \Pi_{1}(w) \Pi_{2}\left(\frac{1}{w}\right) e^{h_{1}(w)}+w_{1} .
\end{aligned}
$$

If $h_{1}(w) \equiv 0$, then $g(z)=C f_{0}(z)+w_{1}$ and $L$ is linear. Hence

$$
\begin{gathered}
f_{0}(z)=D+E g(z)=D+E C f_{0}(z), \\
f(w)=A\left(w-w_{1}\right) e^{E w} .
\end{gathered}
$$

Therefore $E C=1$ and $D=0$. Therefore

$$
\begin{gathered}
f(w)=G\left(w-w_{1}\right) \exp \left(\frac{1}{C}\left(w-w_{1}\right)\right), \\
g(z)=C f_{0}(z)+w_{1} .
\end{gathered}
$$

Thus we have the equivalency of two factorizations. Hence we may assume that $h_{1}(w) \not \equiv 0$. If $h_{1}(w)$ is regular at $w=0$,

$$
m\left(r, \Pi_{2}\left(\frac{1}{w}\right)\right) \sim m\left(r, L\left(h_{2}(w)\right)\right) \sim m\left(r, L\left(\Pi_{2}\left(\frac{1}{w}\right)\right)\right)
$$

as $r \rightarrow 0$. Hence $L$ should be linear. Hence

$$
\begin{aligned}
\Pi_{1}(w) \Pi_{2}\left(\frac{1}{w}\right) & =D+h_{1}(w)+E\left(h_{2}(w)\right) \\
& =D+h_{1}(w)+E C \Pi_{1}(w) \Pi_{2}\left(\frac{1}{w}\right) e^{h_{1}(w)}+w_{1} E
\end{aligned}
$$

Therefore 


$$
f_{0}(z)\left(1-E C e^{M(z)}\right)=D+w_{1} E+M(z) .
$$

This means that $f_{0}(z)$ is a composite function. This is impossible. If $h_{1}(w)$ has a pole at $w=0$, by its construction

$$
m\left(r, \Pi_{2}\left(\frac{1}{w}\right)\right)=o\left(m\left(r, e^{h_{1}(w)}\right)\right)
$$

for $r \rightarrow 0$. On the other hand

$$
\begin{array}{r}
m\left(r, \Pi_{2}\left(\frac{1}{w}\right)\right) \sim m\left(r, L\left(h_{2}(w)\right)\right) \\
\geqq m\left(r, h_{2}(w)\right) \sim m\left(r, e^{h_{1}(w)}\right)
\end{array}
$$

for $r \rightarrow 0$. This is impossible. The same holds when $h_{1}(w)$ has an essential singularity at $w=0$.

The remaining several cases can be treated very easily and lead us to a contradiction.

4. Proof of Theorem 4. Let $F(z)$ be $f(g(z))$. Assume that $f$ and $g$ are transcendental entire. In this case $f$ has only finitely many simple zeros and hence

$$
\begin{gathered}
f(w)=P(w) e^{L(w)} \\
P(w)=A\left(w-w_{1}\right) \cdots\left(w-w_{n}\right) .
\end{gathered}
$$

If there are at least two different zeros of $P(w)$, then $g(z)$ is of order one and hence

Therefore

$$
P(g(z))=B\left(e^{z}-1\right) e^{\alpha z}
$$

$$
\begin{aligned}
e_{n}(z)+e^{-z}+\left(e^{z}-1\right) \exp \left(e_{n}(z)+e^{-z}\right) \\
=D+\alpha z+L(g(z)) .
\end{aligned}
$$

Let $2 p_{1} \pi \imath$ and $2 p_{2} \pi \imath$ be two roots of $g(z)=w_{k}$, then $L(g(z))$ has the same value at these two points. Hence $2\left(p_{1}-p_{2}\right) \pi \imath \alpha$ should be equal to zero. Thus $\alpha=0$. Let us put $w=\exp \left(z / n_{0}\right)$, where $n_{0}$ is defined by $g(z)=h_{2}\left(\exp \left(z / n_{0}\right)\right)$ with a onevalued regular function $h_{2}(w)$ in $0<|w|<\infty$. This is possible by $P(g(z))=B\left(e^{z}-1\right)$. Then

$$
\begin{gathered}
h_{2}(w)=B\left(w^{n_{0}}-1\right), \\
e_{n-1}\left(w^{n_{0}}\right)+\frac{1}{w^{n_{0}}}+\left(w^{n_{0}}-1\right) \exp \left(e_{n-1}\left(w^{n_{0}}\right)+\frac{1}{w^{n_{0}}}\right) \\
=D+L\left(h_{2}(w)\right) .
\end{gathered}
$$


$h_{2}(w)$ is regular at $w=0$. This is impossible. If there is only one zero of $P(w)$, then

This gives

$$
\begin{gathered}
f(w)=A\left(w-w_{1}\right) e^{L(w),} \\
A\left(g(z)-w_{1}\right)=B\left(e^{z}-1\right) e^{M(z)} .
\end{gathered}
$$

$$
\begin{aligned}
e_{n}(z)+e^{-z}+\left(e^{z}-1\right) \exp \left(e_{n}(z)+e^{-z}\right) \\
=D+M(z)+L(g(z)) .
\end{aligned}
$$

In this case $M(z)$ satisfies $M(z+2 \pi i)=M(z)$ as in [9]. If $M(z) \equiv 0$, we have a contradiction easily. Let us put $M(z)=h_{1}\left(e^{z}\right)$ and $g(z)=h_{2}\left(e^{z}\right)$ with one-valued regular functions $h_{1}(w), h_{2}(w)$ in $0<|w|<\infty$. Let us put $w=e^{z}$. Then

$$
\begin{gathered}
h_{2}(w)-w_{1}=C(w-1) e^{h_{1}(w)}, \\
e_{n-1}(w)+\frac{1}{w}+(w-1) \exp \left(e_{n-1}(w)+\frac{1}{w}\right) \\
=D+h_{1}(w)+L\left(h_{2}(w)\right) .
\end{gathered}
$$

Evidently $h_{1}(w)$ is not regular at $w=0$. If $h_{1}(w)$ has an essential singularity at $w=0$,

$$
\begin{aligned}
& m\left(r, \exp \frac{1}{w}\right) \sim m\left(r, L\left(e^{h_{1}(w)}\right)\right) \\
& \quad \geqq m\left(r, e^{h_{1}(w)}\right) \geqq K m\left(r, \exp \frac{1}{w}\right)
\end{aligned}
$$

for an arbitrary $K>1$ as $r \rightarrow 0$. This is a contradiction. If $h_{1}(w)$ has a multiple pole at $w=0$, we have a contradiction similarly. Hence $h_{1}(w)$ has a simple pole at $w=0$. If $L$ is transcendental, then as $r \rightarrow 0$

$$
\begin{aligned}
\frac{1}{\pi r} & \sim m\left(r, \exp \frac{1}{w}\right) \sim m\left(r, L\left(e^{h_{1}(w)}\right)\right) \\
& \geqq K m\left(r, e^{h_{1}(w)}\right) \sim K m\left(r, e^{|a| / w}\right) \sim K \frac{|a|}{\pi r}
\end{aligned}
$$

for an arbitrary $K>1$. This is impossible. Hence $L$ should be a polynomial. Let $m$ be the degree of $L(w)$. Then

$$
\begin{aligned}
&(w-1) \exp \left(e_{n-1}(w)+\frac{1}{w}\right)+e_{n-1}(w)+\frac{1}{w} \\
&=D+h_{1}(w)+a_{m} C^{m}(w-1)^{m} e^{m h_{1}(w)}+\cdots+a_{0} .
\end{aligned}
$$

Therefore as $w \rightarrow 0$ 


$$
m h_{1}(w)-\frac{1}{w}=\alpha+o(w)
$$

with a constant $\alpha$. Let $h_{1}(w)$ be

$$
\frac{b_{-1}}{w}+b_{0}+b_{1} w+\cdots
$$

around $w=0$. Then $m b_{-1}=1$ and further

$$
L(w)=a_{m} w^{m}+a_{0} .
$$

Since $h_{1}(w)-1 / w$ should be regular at $w=0, m=1, b_{-1}=1$. Thus $L$ should be linear. Hence we have

$$
\begin{aligned}
& e_{n-1}(w)+\frac{1}{w}+(w-1) \exp \left(e_{n-1}(w)+\frac{1}{w}\right) \\
& =D+\frac{1}{w}+h_{3}(w)+E\left(w_{1}+C(w-1) e^{(1 / w)+h_{3}(w)}\right),
\end{aligned}
$$

where $h_{3}(w)$ is an entire function. Thus

$$
\begin{aligned}
e_{n-1}(w) & +(w-1) e^{(1 / w)}\left(\exp e_{n-1}(w)-E C e^{h_{3}(w)}\right) \\
& =D+h_{3}(w)+E w_{1} .
\end{aligned}
$$

In this case

$$
\exp e_{n-1}(w) \equiv E C e^{h_{3}(w)}
$$

and hence

$$
e_{n-1}(w)-h_{3}(w) \equiv \text { const. }
$$

Let us put $\beta=h_{3}(w)-e_{n-1}(w)$. Then

$$
\begin{aligned}
& h_{1}(w)=\frac{1}{w}+e_{n-1}(w)+\beta, \\
& h_{2}(w)=w_{1}+C(w-1) e^{(1 / w)+e_{n-1}(w)+\beta} .
\end{aligned}
$$

Hence

$$
g(z)=w_{1}+C^{\prime}\left(e^{z}-1\right) \exp \left(e_{n-1}\left(e^{z}\right)+e^{-z}\right)
$$

and

$$
f(w)=A^{\prime}\left(w-w_{1}\right) e^{E\left(w-w_{1}\right)} .
$$

Thus we have the desired equivalency of two factorizations. The remaining several cases are easily rejected.

5. Proof of Theorem 5. Firstly it is necessary to show the primeness of $f_{0}(w)$. Let us put $f_{0}(w)=f(g(w))$. Assume that $f$ is transcendental meromorphic 
and $g$ is transcendental entire. Then $f$ is of order zero by Edrei-Fuchs' theorem [3]. Further $g$ is of order zero. Let us put $f(x)=f_{1}(x) / f_{2}(x) . \quad f_{1}$ and $f_{2}$ have infinitely many zeros $\left\{v_{j}\right\}$ and $\left\{w_{j}\right\}$, respectively. In this case it is easy to show the following fact: Once $g(w)=v$, has a simple root it only has simple roots. Hence, if $g(w)=v$, has a multiple root, then all the roots are multiple roots, whose orders are not less than $\nu_{1} \geqq 3$. Therefore there is at most one $v_{\text {, for }}$ which $g(w)=v$, has a multiple root. The same holds for $g(w)=w_{\jmath}$. Further either $g(w)=v$, or $g(w)=w$, has only simple roots for every $\jmath$. We may assume that the former case occurs. Let $v_{j 1}, \cdots, v_{j N}$ be the subset of $\left\{v_{j}\right\}$ for which every root of $g(w)=v_{\text {ss }}$ as a root of $f_{0}\left(g_{0}(w)\right)=0$ has its order $\nu_{\jmath}$. Then

$$
\left(1-\frac{w}{a_{\jmath}}\right)^{\nu \jmath}=A \prod_{k=1}^{N}\left(1-\frac{g(w)}{v_{j k}}\right)^{\nu,},
$$

that is,

$$
1-\frac{w}{a_{\jmath}}=A^{\prime} \prod_{k=1}^{N}\left(1-\frac{g(w)}{v_{\jmath k}}\right) .
$$

This is evidently a contradiction, unless $N=1$ and $g(w)$ is linear. If $g$ is a polynomial of degree $n \geqq 2$, the set of zeros of $f_{0}(w)$ should be asymptotically $n$-ply symmetric up to their multiplicities around the point at infinity. However this is not the case. If $f$ is a polynomial or a rational function, then the set of zeros and poles of $f(g(w))$ cannot cover all the zeros and poles of $f_{0}(w)$. Thus $f_{0}(w)$ is prime.

From now on we shall prove the uniqueness of the given factorization $f_{0}\left(g_{0}(z)\right)$. Let $f_{0}\left(g_{0}(z)\right)$ be $f(g(z))$. Since $f_{0}\left(g_{0}(z)\right)$ is of order less than one, $f$ is of order zero if $g$ transcendental. Assume that $f$ is transcendental meromorphic and $g$ is transcendental entire. As in the above process we have

$$
\prod_{k=1}^{N}\left(1-\frac{g(z)}{v_{j k}}\right)^{\nu}=A\left(1-\frac{g_{0}(z)}{a_{j}}\right)^{\nu},
$$

that is,

$$
A^{\prime}\left(1-\frac{g_{0}(z)}{a_{\jmath}}\right)=\prod_{k=1}^{N}\left(1-\frac{g(z)}{v_{j k}}\right)
$$

Since $g_{0}(z)$ is prime, $N$ should be equal to one. Thus $g=C g_{0}+D$ and $f_{0}\left(g_{0}\right)=f(g)$ $=f\left(C g_{0}+D\right)$. Let us put $w=g_{0}$. Then $f_{0}(w)=f(C w+D)$. This gives the desired unicity. The remaining cases can be handled very easily and we have a contradiction.

6. Proof of Theorem 6. Let $F(z)$ be $f(g(z))$.

Case 1). $f$ and $g$ are transcendental entire. (a). Suppose that $f(w)$ has infinitely many zeros $\left\{w_{n}\right\}_{n=1}^{\infty}$. Let us consider $g(z)=w_{n}$. Then all the roots of $g(z)=w_{n}$ for all $n$ lie in the given sector. Further by the second main theorem 


$$
\begin{aligned}
K m(r, g) & \leqq \sum_{n=1}^{\infty} N\left(r, w_{n}, g\right) \\
& =N\left(r, 0, g_{1}\right) \leqq m\left(r, g_{1}\right)
\end{aligned}
$$

for an arbitrary constant $K$ and for $r \notin E$, whose measure is finite. Thus the order $\rho_{g}$ of $g$ satisfies $\rho_{g} \leqq \rho$. If $\rho_{g} \geqq 1$, then by Lemma 5 in [8] the opening $\phi$ of the complementary sector of the given sector should satisfy $\phi \leqq 2 \pi-\pi / \rho_{\mathrm{g}}$ but $2 \pi-\pi / \rho_{g}<2 \pi-\pi /\left(\rho_{g}+\varepsilon\right) \leqq 2 \pi-\pi /(\rho+\varepsilon)=\phi$. This is a contradiction. If $\rho_{g}<1$, then

for $\rho \geqq 1$ and

$$
|\pi-\arg z| \leqq \frac{\pi}{2(\rho+\varepsilon)} \leqq \frac{\pi}{2(1+\varepsilon)}
$$

$$
|\pi-\arg z| \leqq \frac{\pi}{2(1+\varepsilon)}
$$

for $\rho<1$. Hence by Lemma 6 in [8] $g(z)$ should be linear. This is a contradiction.

The remaining cases can be discussed as in the proof of Theorem 1 in [8]. So we omit its details.

7. The following function

$$
F(z)=\left(e^{z}-1\right) \exp \left\{e^{z}-2 z+\left(e^{z}-1\right) \exp \left(e^{z}-2 z\right)\right\}
$$

is a little bit interesting. This function admits

$$
\left(w e^{w}\right) \circ\left\{\left(e^{z}-1\right) \exp \left(e^{z}-2 z\right)\right\} \text {. }
$$

The right factor is E-prime [5] but not prime, since it admits

$$
\left(\frac{w-1}{w^{2}} e^{w}\right) \circ e^{z} \text {. }
$$

Firstly we shall prove the unique factorizability of $F(z)$. Let $F(z)$ be $f(g(z))$. Assume that $f$ and $g$ are trascendental entire. Then $f$ has only finitely many zeros. If $f$ has at least two different zeros, then $g$ should be of order one. Hence

$$
\begin{gathered}
f(w)=P(w) e^{L(w)}, \\
P(g(z))=A\left(e^{z}-1\right) e^{\alpha z}, \\
L(g(z))+\alpha z+D=e^{z}-2 z+\left(e^{z}-1\right) e^{e^{z}-2 z} .
\end{gathered}
$$

Let $g\left(2 p_{1} \pi i\right)$ and $g\left(2 p_{2} \pi i\right)$ be equal to $w_{1}$ such that $f\left(w_{1}\right)=0$. Then $\alpha=-2$. Hence

$$
\begin{gathered}
P(g(z))=A\left(e^{z}-1\right) e^{-2 z} \\
L(g(z))+D=e^{z}+\left(e^{z}-1\right) e^{e^{z}-2 z}
\end{gathered}
$$


In this case $g(z)$ is periodic with period $2 n_{0} \pi i$. Hence

$$
g(z)=h_{1}\left(e^{z / n_{0}}\right)
$$

with a one-valued regular function $h_{1}(w)$ in $0<|w|<\infty$. Let us put $W=e^{z / n_{0}}$. Then

$$
\begin{gathered}
P\left(h_{1}(w)\right)=A\left(W^{n_{0}}-1\right) W^{-2 n_{0}}, \\
L\left(h_{1}(W)\right)+D=W^{n_{0}}+\frac{W^{n_{0}}-1}{W^{2 n_{0}}} e^{W^{n_{0}}} .
\end{gathered}
$$

$h_{1}(W)$ has a pole at $W=0$. Hence $L$ should be a polynomial. Further $h_{1}(W)$ is regular at $W=\infty$. Hence $L\left(h_{1}(W)\right)$ is regular at $W=\infty$. This is a contradiction. If $f(w)=0$ has only one root $w=w_{1}$, which must be simple, then

$$
\begin{gathered}
f(w)=A\left(w-w_{1}\right) e^{L(w)}, L\left(w_{1}\right)=0 \\
A\left(g(z)-w_{1}\right)=B\left(e^{z}-1\right) e^{M(z)}, M(0)=0 \\
L(g(z))+M(z)+D=e^{z}-2 z+\left(e^{z}-1\right) \exp \left(e^{z}-2 z\right) .
\end{gathered}
$$

Let $N(z)$ be $M(z+2 \pi i)-M(z)$. If $N(z) \equiv \equiv$ const,

$$
g(z+2 \pi i)-g(z)=C\left(e^{z}-1\right) e^{M(z)}\left(e^{N(z)}-1\right)
$$

and

imply that

$$
L(g(z+2 \pi i))-L(g(z))=-N(z)-4 \pi \imath
$$

$$
\bar{N}\left(r, 1, e^{N}\right) \leqq N(r,-4 \pi \imath, N(z)) .
$$

This gives a contradiction. Hence $N(z)$ reduces to a constant. If $N(z) \equiv C \neq 2 p \pi i$, then

$$
0=L(g(2 \pi i))-L(g(0))=-C-4 \pi \imath .
$$

This is a contradiction. If $N(z) \equiv 2 p \pi i$, then $g(z+2 \pi i)=g(z)$ and $0=L(g(z+2 \pi i)$ $-L(g(z))=-2 p \pi i-4 \pi i$. Thus $p=-2$. Hence $M(z)=-2 z+h_{1}\left(e^{z}\right)$ and $g(z)=h_{2}\left(e^{z}\right)$ $-w_{1}$ with one-valued regular functions $h_{1}(w), h_{2}(w)$ in $0<|w|<\infty$. Let $W$ be $e^{z}$. Then

$$
\begin{gathered}
-w_{1}+h_{2}(W)=C(W-1) \frac{e^{h_{1}(W)}}{W^{2}}, \\
L\left(h_{2}(W)\right)+h_{1}(W)+D=W+\frac{W-1}{W^{2}} e^{W} .
\end{gathered}
$$

This shows that $h_{1}(W)$ is regular at $W=0$. Hence $h_{2}(W)$ has a double pole at $W=0$. This implies that $L$ is linear. Let $L(w)=E\left(w-w_{1}\right)$. Thus

$$
E C(W-1) \frac{e^{h_{1}(W)}}{W^{2}}+h_{1}(W)+D=W+\frac{W-1}{W^{2}} e^{W} .
$$


Hence $h_{1}(W)=\alpha W+\beta$. This implies that

$$
E C e^{\beta}=1, \alpha=1, \beta+D=0, \beta+\alpha=0 .
$$

Therefore $\log B+2 q \pi \imath=D=1$ show that $B=e, q=0$. Thus

$$
\begin{gathered}
-w_{1}+g(z)=\frac{1}{E}\left(e^{z}-1\right) e^{e^{z}-2 z}, \\
f(w)=E\left(w-w_{1}\right) e^{E\left(w-w_{1}\right)} .
\end{gathered}
$$

This gives the equivalency of two factorizations. Assume that $f$ is transcendental meromorphic and $g$ is transcendental entire. Then

$$
\begin{gathered}
f(w)=\frac{f^{*}(w)}{\left(w-w_{0}\right)^{n}}, \quad f^{*}\left(w_{0}\right) \neq 0, \quad f^{*}(w)=P(w) e^{L(w),} \\
g(z)-w_{0}=A e^{M(z)}, \quad M(0)=0,
\end{gathered}
$$

where $P(w)$ is a polynomial. In this case, if $P(w)$ has at least two zeros,

$$
m\left(r, e^{M}\right) \sim m(r, g)
$$

is of order one. Hence $M(z)=\alpha z$ and

$$
P(g(z))=B\left(e^{z}-1\right) e^{\beta z} .
$$

Further

$$
L(g(z))+D=(n \alpha-\beta-2) z+e^{z}+\left(e^{z}-1\right) \exp \left(e^{z}-2 z\right) .
$$

We put $z=2 p_{1} \pi \imath$ and $z=2 p_{2} \pi \imath$ such that $g\left(2 p_{1} \pi i\right)=g\left(2 p_{2} \pi i\right)=w_{1}, f\left(w_{1}\right)=0$. Then we have $n \alpha-\beta-2=0$. By two equations

$$
\begin{gathered}
g(z)-w_{0}=A e^{\alpha z}, \\
P_{1}\left(g(z)-w_{0}\right)=P(g(z))=B\left(e^{z}-1\right) e^{\beta z},
\end{gathered}
$$

we have

$$
a_{m} A^{m} e^{m \alpha z}+\cdots+a_{0}=B e^{(\beta+1) z}-B e^{\beta z} .
$$

Case 1). $\quad \beta=0$. Then $m \alpha=1, a_{m} A^{m}=B, a_{j}=0(\jmath=1, \cdots, m-1), a_{0}=-B$. Hence $\alpha=1 / m$ and $\alpha=2 / n$. Thus

$$
\begin{gathered}
g(z)-w_{0}=A e^{z / m}, \\
L(g(z))+D=e^{z}+\left(e^{z}-1\right) \exp \left(e^{z}-2 z\right) .
\end{gathered}
$$

Let us put $w=\exp (z / m)$. Then

$$
L\left(w_{0}+A w\right)+D=w^{m}+\frac{w^{m}-1}{w^{2 m}} e^{w^{m}} .
$$

This is evidently absurd. 
Case 2). $\beta+1=0$. Then $\beta=m \alpha$. This gives $\alpha=1 / n$ and $\alpha=-1 / m$. Since $m, n$ are positive integers, we have a contradiction.

Case 3$) . \quad \beta \neq 0, \beta+1 \neq 0$. Then two subcases may occur.
i) $\left\{\begin{array}{l}\beta+1=m \alpha, \\ \beta=q \alpha\end{array}\right.$
ii) $\left\{\begin{array}{l}\beta=m \alpha \\ \beta+1=q \alpha\end{array}\right.$

with $1 \leqq q \leqq m-1$. In these two subcases we have again a contradiction by $n \alpha=\beta+2$.

If $P(w)$ is linear, then

$$
g(z)-w_{1}=C\left(e^{z}-1\right) e^{N(z)} .
$$

We already have

$$
g(z)-w_{0}=A e^{M(z)} .
$$

Then we have two subcases

$$
\text { i) }\left\{\begin{array} { l } 
{ M ( z ) = z } \\
{ N ( z ) = 0 }
\end{array} \text { ii) } \left\{\begin{array}{l}
M(z)=-z \\
N(z)=-z
\end{array}\right.\right.
$$

In the former case

$$
g(z)-w_{0}=A e^{z} \quad \text { or } \quad g(z)-w_{1}=C\left(e^{z}-1\right)
$$

and

$$
\begin{aligned}
L(g(z)) & +N(z)-n M(z)+D \\
= & e^{z}-2 z+\left(e^{z}-1\right) \exp \left(e^{z}-2 z\right) .
\end{aligned}
$$

Then $N(z)-n M(z)=-n z=-2 z$. Hence

$$
L(g(z))+D=e^{z}+\left(e^{z}-1\right) \exp \left(e^{z}-2 z\right) .
$$

Let us put $w=e^{z}$. Then

$$
L\left(w_{0}+A w\right)+D=w+\frac{w-1}{w^{2}} e^{w} .
$$

This gives an absurdity. In the latter case

$$
g(z)-w_{0}=A e^{-z} \text { or } \quad g(z)-w_{1}=C-C e^{-z}
$$

and

$$
L(g(z))+D=-(n+1) z+e^{z}+\left(e^{z}-1\right) \exp \left(e^{z}-2 z\right) .
$$

In this case firstly we have $n+1=0$ and

$$
L\left(w_{0}+\frac{A}{w}\right)+D=w+\frac{w-1}{w^{2}} e^{w} .
$$


This implies that $L$ is a polynomial. The left hand side is regular at $w=\infty$ but the right hand side is not. This is impossible. Assume that $f$ is a polynomial and $g$ is transcendental. In this case $g$ must be entire. If $f$ has two different zeros, then $g$ should be of order one and the same holds for $f(g(z))$. This is clearly absurd. Since $f$ has no multiple zero, $f$ is linear. We have nothing to prove. Assume that $f$ is transcendental and $g$ is a polynomial. In this case $f$ must be entire. By the distribution of zeros of $F, g$ should be of degree at most two. If $g$ is really quadratic, then the distribution of values of $f(g(z))$ should be asymptotically symmetric. However $F(z)$ does not satisfy this symmetricity. Assume that $f$ is rational and $g$ is entire. Then

$$
\begin{gathered}
f(w)=\frac{P(w)}{\left(w-w_{0}\right)^{n}}, \quad P\left(w_{0}\right) \neq 0, \\
P(g(z))=A\left(e^{z}-1\right) e^{M(z)}, \quad M(0)=0, \\
g(z)-w_{0}=B e^{N(z)}, \quad N(0)=0 .
\end{gathered}
$$

Here $P(w)$ is a polynomial. Case 1$). \quad P(w)$ is linear. Then

$$
g(z)-w_{1}=C\left(e^{z}-1\right) e^{M(z)} .
$$

Hence we have either $M(z) \equiv 0, N(z)=z$ or $M(z)=-z=N(z)$. Then in both cases $g(z)$ is of order one, which gives clearly a contradiction. Case ii). $P(w)$ is not linear. Since $P(w)$ has no multiple zero, there are at least two different zeros. Then $g(z)$ is of order one, which is again impossible. Assume that $f$ is rational and $g$ is meromorphic. Let $a_{1}$ be a pole of $f$. Then $g(z)-a_{1} \neq 0$. Let $g_{1}$ be $1 /\left(g-a_{1}\right)$. Then $F(z)=R\left(g_{1}(z)\right)$ with a rational function $R$ and entire $g_{1}(z)$. Thus this case reduces to the above case.

Therefore we have the desired result.

As already remarked we have

with

$$
F(z)=f_{1}\left(g_{1}(z)\right)
$$

and

$$
f_{1}=W e^{W}, g_{1}=\left(e^{z}-1\right) \exp \left(e^{z}-2 z\right)
$$

$$
g_{1}(z)=\left\{\frac{X-1}{X^{2}} e^{X}\right\} \circ e^{z} \equiv f_{2}\left(e^{z}\right)
$$

Hence

$$
F(z)=f_{1} \circ\left\{f_{2} \circ e^{z}\right\}
$$

However, since $f_{1} \circ f_{2}$ has an essential singularity at the origin,

$$
F(z)=\left\{f_{1} \circ f_{2}\right\} \circ e^{z}
$$

does not hold if we do not admit any factorization in the extended sense. This is a little bit inconvenience, since the associative law has been destroyed. In 
order to secure the associative law it is necessary to extend the class of admissible functions. A method to do it is the following: $f_{1} \circ f_{2}$ is admissible if $f_{2}$ is one-valued regular in $0<|z|<\infty$, even if $f_{1}$ is transcendental entire. This method of extension gives us a new inconvenience, since every periodic entire function is not prime by this extension. Thus we gain an advantage together with a disadvantage.

\section{REFERENCE}

[1] Baker, I.N. And F. Gross, On factorizing entire functions. Proc. London Math. Soc. 13 (1968), 69-76.

[2] EDREI, A. Meromorphic functions with three radially distributed values. Trans. Amer. Math. Soc. 78 (1955), 276-293.

[3] Edrei, A. AND W. H. J. Fuchs, On the zeros of $f(g(z))$ where $f$ and $g$ are entire functions. J. Analyse Math. 12 (1964), 243-255.

[4] Kobayashi, T. On a characteristic property of the exponential function. Kōda1 Math. Sem. Rep. 29 (1977), 130-156.

[5] Ozawa, M. Factorization of entire functions. Tôhoku Math. J. 27 (1975), 321336.

[6] Ozawa, M. On certain criterıa for the left-primeness of entire functions. Kōdai Math. Sem. Rep. 26 (1975), 304-317.

[7] Ozawa, M. On certain criteria for the left-primeness of entıre functions., II. Kōdai Math. Sem. Rep. 27 (1976), 1-10.

[8] Ozawa, M. On uniquely factorizable entire functions. Kōdai Math. Sem. Rep. 28 (1977), 342-360.

[9] Ozawa, M. On the exıstence of prıme periodic entıre functıons. Kōdaı Math. Sem. Rep. 29 (1978), 308-321.

[10] Rényi, A. AND C. RÉnyi, Some remarks on periodic entire functions. J. Analyse Math. 14 (1965), 303-310.

Department of Mathematics,

TOKyo Institute of TECHNOLOGY

Oh-okayama, Meguro-ku, Tokyo, Japan 